\title{
INFRARED THERMOGRAPHY WITH OPTICAL AND ULTRASONIC EXCITATION: PROMISING TOOLS FOR THE CHARACTERIZATION OF VERTICAL CRACKS
}

\author{
A. Mendioroz ${ }^{1+*}$, R. Celorrio ${ }^{2}$, A. Salazar ${ }^{1}$ \\ ${ }^{1}$ Departamento de Física Aplicada I, Escuela Técnica Superior de Ingeniería, Universidad del País Vasco UPV/EHU, \\ Alameda Urquijo s/n, 48013 Bilbao, Spain \\ ${ }^{2}$ Departamento de Matemática Aplicada, EINA/IUMA, Universidad de Zaragoza, Campus Río Ebro, Edificio Torres \\ Quevedo, 50018 Zaragoza, Spain \\ †*arantza.mendioroz@ehu.eus
}

\begin{abstract}
Active infrared thermography is nowadays recognized as an efficient nondestructive evaluation tool to detect defects such as cracks, delaminations, or voids in a wide variety of materials. In this presentation we will focus on the characterization of vertical cracks using optical and ultrasonic excitation of the sample, as complementary techniques for open and kissing cracks, respectively. We will show that, for open cracks, optically excited thermography with a focused illumination is very well suited to characterize the thermal resistance (opening) and size of open vertical cracks. We present analytical calculations of the surface temperature distribution produced by infinite cracks, we analyze the optimum experimental conditions to characterize them and we point out a new methodology to deal with finite and thin cracks, based on discontinuous Galerkin finite elements. In the second part, we will show that ultrasound excited thermography is a very appropriate choice to characterize kissing cracks, as the rubbing of the contacting surfaces produces heat at the crack. We will describe the methodology we have developed to retrieve the geometry of the heat source distribution produced at the crack from surface temperature vibrothermography data. Experimental verification of the potential of infrared thermography with optical and ultrasonic excitation to characterize calibrated open and kissing vertical cracks will be presented.
\end{abstract}

KEYWORDS: infrared thermography, vibrothermography, vertical cracks, inverse problems, nondestructive evaluation.

\section{INTRODUCTION}

One of the most extended applications of active infrared thermography (IRT) is the detection of delaminations, typically parallel to the sample surface, caused by impact damage in composites, which is usually implemented with homogeneous optical excitation of the surface. On the contrary, in order to detect vertical cracks by optically excited IRT one needs to generate a heat flux across the crack, which is generally achieved by focusing a laser beam on one side of the crack. Open cracks act as a thermal resistance that hinders heat propagation and produce an asymmetry in the temperature across the crack, which is the signature of the presence of the crack. However, very narrow or kissing cracks may remain unnoticed as they produce very small thermal resistances. These kissing cracks are target defects for ultrasound excited thermography (or vibrothermography) as the relative vibration of the contacting faces produces heat, and the crack turns into a heat source in the presence of ultrasounds, so it is revealed as a hot spot at the surface. In this work we present our recent advances on the characterization of vertical open and kissing cracks using optical and ultrasound excited IRT, respectively.

\section{FOCUSED OPTICAL EXCITATION: OPEN CRACKS}

If we consider a material that contains an infinite vertical crack, the calculation of the temperature distribution at the surface when it is illuminated by a modulated gaussian laser beam can be done analytically [1]. We analyze the temperature profile perpendicular to the crack through the center of the laser spot to determine the optimum experimental conditions to characterize the thermal resistance produced by the crack, which is related to the opening of the crack. The experiments performed with artificial open calibrated cracks of different widths (Fig. 1) confirm that it is possible to characterize the width of the crack from focused optically excited thermography. However, if the crack is not infinite, there is no analytical solution for the surface temperature distribution and the calculation has to be carried out numerically. Regular finite element modeling fails to describe thin cracks because the mesh of the air gap filling the crack needs to be extremely fine. We have developed discontinuous Galerkin finite elements in which the crack is described as an interface characterized by a thermal resistance, that allow us to 
calculate the surface temperature produced by cracks of any shape and size, including very thin cracks [2]. Calculations and experiments show that when the crack lips are in contact, the surface temperature signature is barely visible.
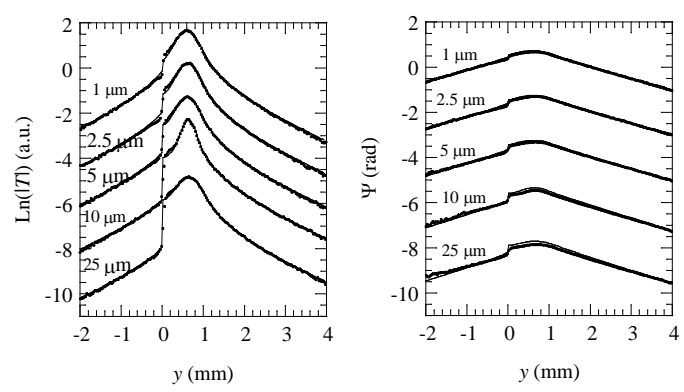

Fig. 1 Experimental surface temperature profiles (symbols) perpendicular to infinite calibrated cracks, through the center of the laser spot, and fittings to the analytical model (continuous lines).

\section{VIBROTHERMOGRAPHY: KISSING CRACKS}

Kissing cracks behave like heat sources in vibrothermography experiments. We have obtained the semi-analytical expressions for the temperature distribution produced by vertical heat sources of any shape excited by an ultrasound burst. The characterization of these heat sources from vibrothermography experiments involves solving the inverse problem which consists in retrieving the heat source distribution responsible for the observed surface temperature. This is an ill-posed inverse problem which means that the minimization of the residual is unstable. We present the stabilization techniques we have developed [3] to characterize vertical heat sources from vibrothermography experiments (see Fig. 2).

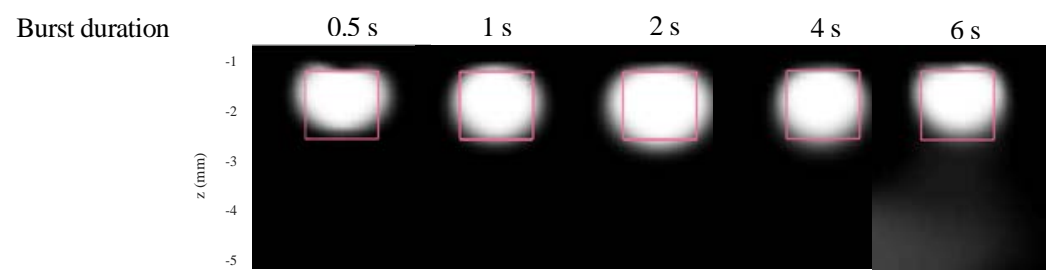

Fig. 2 Gray level representation of the heat source distribution obtained from experimental data corresponding to calibrated homogeneous heat sources (delimited by the red contours), for different burst durations. White represents maximum value of the retrieved heat flux and black is absence of heat sources.

\section{CONCLUSIONS}

Optical and ultrasound excited IRT are complementary efficient tools for the characterization of vertical open and kissing cracks respectively. The characterization of cracks of any shape needs sophisticated inversion algorithms to deal with the ill-posedness of the inverse problem.

\section{ACKNOWLEDGMENT}

This work has been supported by Ministerio de Economía y Competitividad, (DPI2016-77719-R and MTM2016-75139-R, AEI/FEDER, UE), by Gobierno Vasco (KK-2016/00027) and by Universidad del País Vasco UPV/EHU (GIU16/33).

\section{REFERENCES}

[1] N.W. Pech-May, A. Oleaga, A. Mendioroz, A. J. Omella, R. Celorrio, A. Salazar, Vertical cracks characterization using lock-in thermography: I. Infinite cracks, Meas. Sci. Technol. 24 (2013) 065601 (11pp).

[2] R. Celorrio, A.J. Omella, N.W. Pech-May, A. Oleaga, A. Mendioroz, A. Salazar, Vertical cracks characterization using lock-in thermography: II. Finite cracks, Meas. Sci. Technol. 24 (2013) 065602 (9pp).

[3] A. Mendioroz, R. Celorrio, A. Cifuentes, L. Zatón, A. Salazar, Sizing vertical cracks using burst vibrothermography, NDE\&T Int. 84 (2016) 36-46. 